\title{
Artificial Rhythm Recognition using Portable Cardiomonitor and Mobile Application
}

\author{
Maria Chaykovskaya ${ }^{1}$, Alexander Kalinichenko ${ }^{2}$, Ekaterina Fetisova ${ }^{1}$, \\ Sergey Mironovich ${ }^{1}$, Alexey Kiprensky ${ }^{1}$ \\ ${ }^{1}$ Petrovsky National Research Centre of Surgery, Moscow, Russia \\ ${ }^{2}$ Saint Petersburg Electrotechnical University, Russia
}

\begin{abstract}
Patients with implanted pacemakers (PM) are usually not familiar with their pacing modes and other device settings. Modern bipolar endocardial pacing leads produces such a small pacing artifact that often could not be separated from noise. It becomes a challenge to judge about the underlying rhythm and current pacing mode using single ECG tracing. Good stimuli artifacts visualization and understanding of its interaction with QRS plays crucial role in ECG analysis.
\end{abstract}

\section{Introduction}

According to statistic reports overall use of implantable cardiac devices tends to grow in developing countries. In the United States [1] from 1993 to 2009 rate of such interventions increased by 55,6\%. Incidence of dual chamber (DDD) pacemaker (PM) implantation increase in absolute value (from 62\% to 82\%), compared with single chamber devices (AAI or VVI). Same tendency occurs with biventricular (BiV) systems. European Union demonstrates quite similar pattern $[2,3]$ with large geographic heterogeneity between countries due to sociological, economic and epidemiological reasons. This process has a solid base. First, clinical trials, finished last decade, provided evidence for cardiac devices indications expansion. Second - ageing of worldwide population, with increasing rate of primary implantation as well as generator replacement procedures [4]. While patients become older with more comorbidities, PM systems becomes more complex. It takes more efforts from medical stuff to provide adequate care, aimed to improve quality and longevity of life.

ECG remains first and primary method of screening and examination in case of scheduled follow-up visit and in emergency case.

Pacing leads usually have two electrodes, with the location of the electrodes determining polarity of the signal. Due to close position of too electrodes in bipolar models - one on the tip and one ring electrodes, all inside the heart, it provides plenty features, as less impedance and less current use, less interference from extracardiac myopotentials, less chance of diaphragmatic pacing. If the current flows between the tip of the lead and the pulse generator, this is a unipolar pacing mode. And as the current travels through large area of body (with other excitable tissues, which can inhibit or provoke next stimulus) it provides large stimulus artifact. Bipolar stimulus artifact is barely seen because of the small pulse amplitude, needed to capture myocardium in small distance between electrodes (about centimeter).

Second reason of invisibility of the impulses is their width. Very short signals (microseconds) got filtered on a trace with resolution in milliseconds.

Implantable cardioverter-defibrillators (ICD) were developed to recognize life-threatening ventricular arrhythmias and terminate them with high energy shocks, delivering to the heart by endocardial electrodes. All modern ICDs function as PMs, which is useful when heart rhythm is slowed down by prescribed antiarrhythmic drugs.

Very special kind of implanted cardiac devices is cardiac resynchronization therapy (CRT). In these systems too electrodes in each of ventricles by simultaneously (or with little programmed delay) pacing provide its synchronous contraction aiming to improve cardiac output. Vast majority of CRTs have defibrillating function (CRT-D) because these patients meet all criteria to ICD implantation as well. This is the most complex device type, conceived to provide at least $95 \%$ of paced rhythm (VVI or DDD).

The third reason of poor displaying feasibility of stimuli artifacts is the vector of the pulse. Pulses from right atrium are best displayed in lead aVF, right ventricle pulses are best displayed in lead II, if the lead was placed in the apex of right ventricle. Biventricular (BiV) pacing in CRT systems can be very difficult to display properly because two ventricle pulses may occur at the same time, appearing as a single pulse. Left ventricle lead, held 
through coronary sinus, is located on the epicardial surface - outside of the heart. The vector of left ventricle could differ from right ventricle, even orthogonal [5]. Simultaneous pulses with opposite direction could cancel each other on surface ECG. Ventricle leads may be placed in various positions to avoid scar tissue. Modern left ventricle leads have up to 5 electrodes. Changing vector between them and the can of CRT may result in optimal capture.

Patients with implanted cardiac devices could suffer from special arrhythmias, because of device malfunction. It happens basically with unipolar lead systems, because of its sensing and detection features.

In all cases of EGC analyzing in patients with implanted cardiac devices all information about type of device, its pacing mode and other programmable functions should be available. In many situations elderly patients cannot provide this kind of information.

In case of broad complex arrhythmias, PM dependent patients and patients with CRT, stimuli artifacts and QRS shape are useful for differentiation normal paced rhythm from ventricular arrhythmias and PM malfunctions.

\section{Materials and methods}

In our study we enrol 86 consistent patients, hospitalized to Petrovsky National Research Centre of Surgery due to primary cardiac device implantation or generator replacement and patients from out hospital device follow up visits, during February and March 2016. Overall 102 ECG recordings were made under initial parameters and if well tolerated, in other temporary programmed modes. According to global tendency the majority of recordings we made in DDD mode $n=62$ (60,9\%), in VVI $n=23$ (22,5\%), AAI $n=17$ (16,6\%). We had the whole spectrum of cardiac pacing devices: PMs $(n=35,40,7 \%)$, ICDs $(n=31,36,0 \%)$, CRT $n=2,2,3 \%$ and CTR-D $n=18,21,0 \%$. In CTR patients, modes with ventricular stimulation are determined by $\mathrm{BiV}$ stimulation: DDD BiV, n=20 (19,6\%); VVI BiV, n=4 (3,9\%). In 1 patient we temporary shift BiV DDD stimulation to standard right ventricular stimulation in DDD mode.

For ECG recording we used novel portable cardiomonitor: a removable iPhone (5/5S) case with builtin electrodes and an application with original algorithm (CardioQVARK, Moscow, Russia). ECG signal was recorded between fingers of each hands, and equals to I lead of standard 12-lead ECG.

Cardiomonitor uses highly sensitive wide bandpass capacitive electrodes and special algorithm of stimuli artefact detection. When recording is finished it goes to cloud server with use of any of available mobile or Wi-Fi Internet connection. On the server recording underwent filtration from noise, QRS complex and stimuli artefact recognition. This process is based on primary amplitude and frequency analysis of the signal. Here premature contractions can be detected. The origin of premature contraction - atrium or ventricle - may be determined by the form of QRS complex. Afterwards program make more precise analysis of heart rate variability, using time domain and frequency domain methods. Finally, in few seconds the recording goes back to device with calculated heart rate, heat rate variability parameters and marked premature contractions and PM stimuli artifacts if present.

In our work we focused on recognition of paced rhythm using portable cardiomonitor recordings. During ECG recording patient's cardiac device was connected to device programmer, using telemetry or magnet field on the head of the programmer. The doctor made first recording under initial parameters, exactly notice them from the device memory. Afterwards standard threshold capture test was performed, test for underlying rhythm and some other pacing modes were tested. Each step was marked on appropriate ECG.

Besides patient's application for iPhone enable to make and to review previous ECG recordings, there is a special doctor's application for iPad. Using patient's login and password the doctor or nurse or other authorised medical stuff could add patient's profile and being able to run through all his ECG recordings, with marked new ones.

The aim of our study was: validation of algorithm to recognize and properly mark paced stimuli artifacts on ECG tracing. Two experienced cardiologists independently examined all blinded tracings. In conclusion they judge about the rhythm type - paced or spontaneous, about the paced chamber and probable pacing mode, any seen dysrhythmias and pacing malfunctions if present. Lastly we compare initial settings with modes and events recognized by doctors, using single lead ECG tracing.

\section{Results}

Using novel cardiomonitor paced rhythm (Fig. 1-4) was identified in $100 \%$ of recordings by two independent experts. Interpretation of pacing mode, made by an expert $100 \%$ correlates to initially programmed ones.

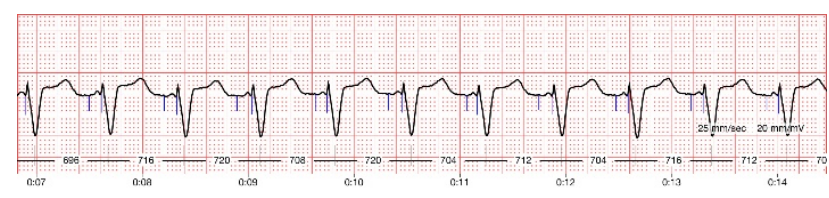

Figure 1. DDD pacing mode, basic rate 85. 


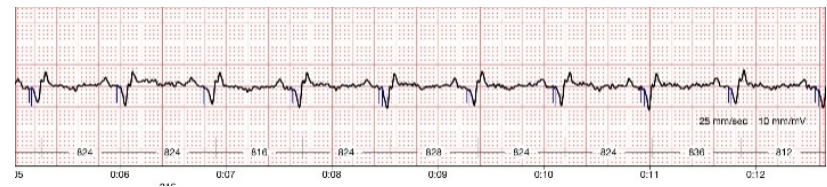

Figure 2. DDD BiV pacing mode: $\mathrm{P}$ sensed biventricular pacing rhythm, 73 beats per minute

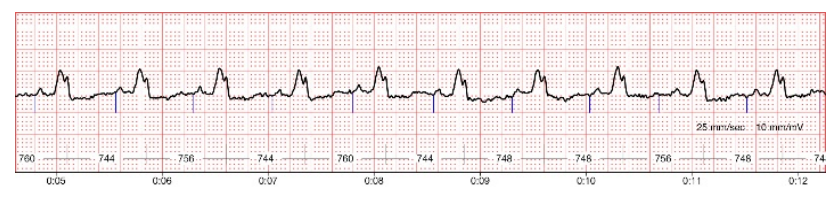

Figure 3. AAI pacing mode, basic rate 80 .

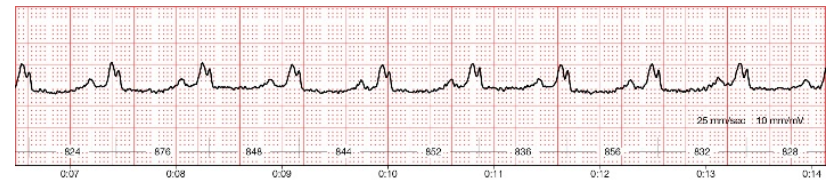

Figure 4. Spontaneous sinus rhythm, 70 beats per minute.

Recognition of modes switch from DDD to VVI or AAI was also very high - 93\%. Episodes of capture loss during threshold testing with shifting to spontaneous rhythm was detected in $84 \%$. Definition of fusion and pseudo fusion beats as well as premature contractions (Fig. 5-6) was absolute (100\%).

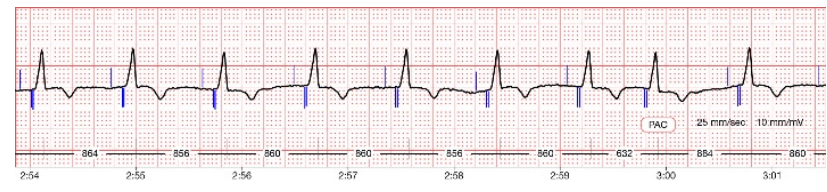

Figure 5. DDD BiV pacing mode, basic rate 80 . Premature atrial contraction (PAC).

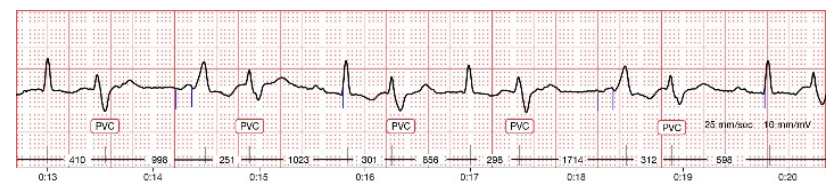

Figure 6. PM malfunction, premature ventricular contractions (PVCs).

\section{Discussion}

Modern life brings necessity of providing healthcare service to people in any time and from anywhere. Elderly people with implanted cardiac devices in case of adversity should visit medical centre or medical stuff should visit them for diagnosis and advising. In any case it takes much from healthcare professional and costs much. We suggest a new approach. Patient plays an active role in illness detection and prevention.

ECG registration on a daily basis or in case of any symptoms allows to collect an individual database of heart activity patterns for each patient. In case of inadequate heart rate or irregular heart beat or nonspecific clinical symptoms ECG recording can make the diagnosis clear.

Pacemaker malfunction can occur for a variety of reasons, from equipment failure to changes in underlying heart condition and native rhythm.

Decision of PM normal function or malfunction might be challenging. It usually takes a number of tests to discover a problem. Real time visualisation of paced stimuli artefacts provides confidence in basic device function - pacing with basic rate. Creating patients profile, except main cardiologic diagnosis and prescribed pills, device type with pacing mode and basic rate are required.

Besides regular resting ECG we have a number of options to use. We can ask the patient to inhale or other vagus maneuvers, to slow down natural heart rhythm. We can ask the patient to exercise and to test the ability to increase natural or paced heart rate. Patient could perform a magnet test. Clear understanding of device response to these tests and its interaction with spontaneous cardiac rhythm allows to determine the type of pacing therapy being administered to the patient.

In case of CRT systems, as listed above, it is crucial to obtain as much pacing as possible. There are about 30\% [6] of "non-responders" to resynchronisation therapy - patients which do not benefit from CRT in heart pumping capacity. A number of conditions may potentiate this unfortunate situation. Like frequent premature contractions, high rate of underling rhythm - in atrial fibrillation, with absence of ventricular pacing or with fusion beats. Left ventricular lead malfunction may be the course of right ventricular pacing. All these situations reflect QRS morphology. Using standard 12lead ESG to achieve typical morphology of biventricular paced QRS, afterwards it becomes possible distantly to compare each day QRS morphologies (Fig. 7). Good pacing artefacts visualization and QRS analysis makes possible to define absence from ineffectiveness of left ventricular component in biventricular pacing. Registration of any other morphology often should be the reason of more precise examination and elimination of the problem - drug therapy or pacing parameters correction. 


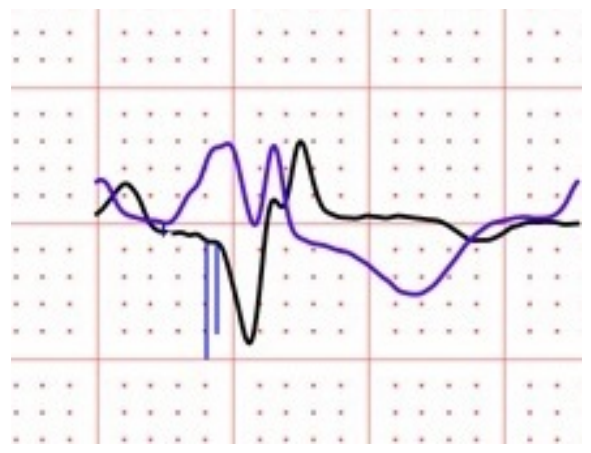

Figure 7. ORS morphology comparison - paced and sensed one.

\section{Conclusion}

Single lead ECG recording with well-visualized stimuli artefacts allows a doctor properly to define intrinsic rhythm and all events, recorded by cardiomonitor.

\section{Acknowledgment}

This work has been supported by the following projects: the Russian Foundation for Basic Research project \#16-07-00722; the Ministry of Education and Science of Russia project \# 14.578.21.0122; medical project "CardioQVARK. ECG by Smartphone" (www.cardioqvark.ru).

\section{References}

[1] Greenspon AJ, Patel JD, Lau E, et al. Trends in Permanent Pacemaker Implantation in the United States From 1993 to 2009: Increasing Complexity of Patients and Procedures. J Am Coll Cardiol. 2012;60(16):1540-1545. doi:10.1016/j.jacc.2012.07.017.

[2] Raatikainen MJ, Arnar DO, Zeppenfeld K et al. Statistics on the use of cardiac electronic devices and electrophysiological procedures in the European Society of Cardiology countries: 2014 report from the European Heart Rhythm Association. Europace. 2015;17 Suppl 1:i1-75. doi: 10.1093/europace/euu300.

[3] Valzania C, Torbica A Tarricone R. et al. Implant rates of cardiac implantable electrical devices in Europe: A systematic literature review. Health Policy, Volume 120 , Issue 1, 1- 15

[4] Bradshaw PJ, Stobie P, Knuiman MW, et al. Trends in the incidence and prevalence of cardiac pacemaker insertions in an ageing population. Open Heart 2014;1:e000177. doi:10.1136/openhrt-2014-000177

[5] Kruse J, Redmond K. Detecting and distinguishing cardiac pacing artifacts. Analog Dialogue 2012; 46-11

[6] Auricchio A, Prinzen FW. Non-responders to cardiac resynchronization therapy: the magnitude of the problem and the issues. Circ J. 2011;75(3):521-7. Epub 2011.

Address for correspondence.

Maria Chaykovskaya

119435, Russia, Moscow, Abrikosovsky pereulok, 2

m.chaykovskaya@list.ru 